\title{
A SIMPLE MASS ESTIMATE FOR CENTRAL BLACK HOLES IN CUSPY GALAXIES
}

\author{
S. DE RIJCKE ${ }^{1}$, V. DE BRUYNE, H. DEJONGHE AND A. MATHIEU \\ Sterrenkundig Observatorium, liniversity of Ghent \\ Krijgslaan 281, S9, B9000 Gent, Belgium
}

\begin{abstract}
We argue that the velocity dispersion of the stars is likely a monotonically decreasing function of radius along the minor axis in the central regions of cuspy galaxies. We show that then a central mass concentration (a black hole) must be present and calculate its minimum mass, $M_{\text {o. }}$ This lower bound is relevant and entirely consistent with current mass estimates.
\end{abstract}

Cusps occur frequently in the density distribution of the central regions of galaxies and bulges. If very massive, compact objects exist, they are most likely to be found in the centers of galaxies. It is a logical thought to associate both phenomena, which is of course a well established idea.

It is possible however to construct dynamical models for the observed kinematics and photometry of cuspy galaxies without the necessity of introducing a black hole $(\mathrm{BH})$, as has been amply demonstrated for M87 and M32. It would then seem that stellar dynamical models hardly constrain the presence of a $\mathrm{BH}$. We impose a restricting postulate on the stellar dynamical models : we assume that the velocity dispersion of the stars is a monotonically decreasing function of galactocentric radius along the minor axis inside the cusp. This assumption is supported both by observational evidence (the velocity dispersion in normal galaxies generally keeps rising inwards at the last measured point) and theoretical arguments (the virial theorem spontaneously produces hot cores).

We approximate the central parts of elliptical galaxies as isotropic and spherically symmetric (the argument also applies to the minor axis of axisymmetric systems). In the absence of a $\mathrm{BH}$, the potential is finite everywhere and the accessible region of phase space is limited. The distribution function (DF) diverges in the center, causing the velocity dispersion to 
become zero for $r \rightarrow 0$. Cuspy galaxies without a BH clearly violate our assumption. Cuspy systems with a $\mathrm{BH}$ have a diverging potential so the accessible region of phase space is unlimited. The DF is finite everywhere. In the center the velocities can be very different and large, causing the velocity dispersion to rise inwards. So only cuspy systems with a BH satisfy our assumption.

For the cuspy spatial density $\rho(r)=\rho_{0} r^{p-2}$, we derive the velocity dispersion $\sigma^{2}(r)=a / r+b r^{2-p}+c r^{p}$, with the coefficients depending on $M_{\bullet}, p, \rho_{0}$ and a radius $R$ inside the cusp for which we know $\sigma^{2}(R)$. Towards the center, the contribution of the BH dominates, $\sigma^{2}(r) \sim 1 / r$, but when the BH mass is too small, farther out in the cusp the velocity dispersion can obviously start rising with radius, which we reject.

The minimum BH mass that causes the velocity dispersion to decrease monotonically with rising $r$ can be obtained by requiring that both the first and second derivative of $\sigma^{2}$ become zero at the same radius $r$. This translates into two equations that can easily be solved iteratively for the radius $r$ and the minimum $M_{\text {. }}$.

In the following table we present some results. When data along the major axis are used, the galaxy is labeled with an asterisk. The presented values are indicative, due to the heterogeneity of the data and the uncertainties on it. Slopes and kinematics are taken from different authors, the method requires deconvolved and deprojected dispersions but usually only projected values are available. When the projected slope was given, we subtracted 1 from it to obtain the deprojected value. The resulting value of $M_{\bullet}$ depends sensitively on $\sigma^{2}(R)$ and the cusp index $p$. The BH masses found in the literature are given in parentheses.

\begin{tabular}{|l|l|}
\hline Galaxy & $M_{\bullet}\left(10^{6} M_{\odot}\right)$ \\
\hline NGC $3377^{*}$ & $2(80)$ \\
M 32 & $1(.7-3)$ \\
M 31* & $3(30)$ \\
NGC $3115^{*}$ & $550(1000)$ \\
NGC 3379 & $140(50)$ \\
NGC 4486B* & $280(500-900)$ \\
M 87* & $2000(1000-5000)$ \\
\hline
\end{tabular}

${ }^{1}$ Research Assistant of the Fund for Scientific Research - Flanders (Belgium)(F.W.O) 